\title{
Boundary Value Problems of Potential Functions in Decagonal Quasicrystals
}

\author{
Wu Li, ${ }^{1}$ Hao Xin, ${ }^{2}$ and Tianyou Fan ${ }^{3}$ \\ ${ }^{1}$ Institute of Applied Mathematics, Taiyuan University of Technology, Taiyuan 030024, China \\ ${ }^{2}$ Institute of Applied Mechanics and Biomedical Engineering, Taiyuan University of Technology, Taiyuan 030024, China \\ ${ }^{3}$ Department of Mathematics, Beijing Institute of Technology, Beijing 100081, China
}

Correspondence should be addressed to Wu Li; liwu823210@126.com

Received 16 April 2014; Revised 29 August 2014; Accepted 30 August 2014; Published 14 October 2014

Academic Editor: Evangelos J. Sapountzakis

Copyright (C) $2014 \mathrm{Wu} \mathrm{Li}$ et al. This is an open access article distributed under the Creative Commons Attribution License, which permits unrestricted use, distribution, and reproduction in any medium, provided the original work is properly cited.

\begin{abstract}
A unified form of potential functions in decagonal quasicrystals (QCs) and conformal mappings are applied in a novel way to solve the boundary value problems emanating from the generalized theory of elasticity for decagonal QCs. By executing the reduction of boundary value problem to function equations, two crack problems are investigated. In the first one, an approximate analysis for bending specimen with a crack is given. In the other, a finite width strip with single edge crack of decagonal QCs is analytically estimated. Using the basic idea underlying Dugdale's crack model, the extent of cohesive force zone in each of the two cases is analytically derived.
\end{abstract}

\section{Introduction}

Soon after the discovery of QCs [1], the boundary value problems of elasticity and defects of QCs have been put forward. In 2011, some scholars found 12-fold and 18-fold diffraction symmetric QCs in colloid, which may arouse great interest [2]. The unusual characters of the QCs are originated from their special atomic constitution. Due to the quasiperiodicity, QCs have a special type of elastic degrees of freedom, termed as phason degrees of freedom apart from phonon degrees which exist too for crystals, which have been studied extensively and widely [3-13]. On the basis of the physical framework and by extending the methodology of mathematical physics and classical elasticity, the mathematical theory of elasticity of QCs has been developed [11, 12, 14, 15]. Recent studies on the elasto-/hydrodynamics of QCs have made preliminary but significant progress $[8,10,13,16-18]$. Wang et al. provide some useful insight into the interface problems of QCs [19]. Feuerbacher et al. have studied plastic deformation of QCs in microscopic mechanism, [20-29]. Li et al. have studied thermoelastic general solutions of one-dimensional hexagonal quasicrystals [30, 31]. More recently, Radi and Mariano investigated the straight cracks and dislocations in two-dimensional QCs by using the Stroh formalism and obtained the closed-form solution to the balance equations in terms of phonon and phason fields $[32,33]$. The Stroh formalism for two-dimensional deformations of the icosahedral QCs was then studied by Li and Liu [34]. Guo et al. have studied a finite crack in a one-dimensional hexagonal quasicrystal strip [35]. As a material, QC is deformable under applied forces, thermal loads, and certain internal effects. The theoretical physicists have proposed various descriptions of its elasticity. The majority agrees that the Landau density wave theory is the physical basis of elasticity of QCs. Essentially, the description suggested that there are two displacement fields $\mathbf{u}$ and $\mathbf{w}$ in a QC: the former is similar to that in crystals, named as the phonon field; the latter is a displacement field, named as the phase field. The total displacement field in a QC is expressed by

$$
\overline{\mathbf{u}}=\mathbf{u}^{\|} \oplus \mathbf{u}^{\perp}=\mathbf{u} \oplus \mathbf{w},
$$

where $\oplus$ denotes the direct sum; $\mathbf{u}$ is in the physical space or the parallel space $E_{\|}^{3} ; \mathbf{w}$ is in the complement space or the perpendicular space $E_{\perp}^{3}$, which is an internal space. Under 
given loading conditions, they are giving rise to two elastic strain tensors $\varepsilon_{i j}$ and $w_{i j}$, respectively [36]:

$$
\varepsilon_{i j}=\frac{1}{2}\left(\frac{\partial u_{i}}{\partial x_{j}}+\frac{\partial u_{j}}{\partial x_{i}}\right), \quad w_{i j}=\frac{\partial w_{i}}{\partial x_{j}}, \quad\left(w_{i j} \neq w_{j i}\right) .
$$

In the case of small deformation of the decagonal QCs, the phonon and phason stresses obey generalized Hooke's law, namely,

$$
\sigma_{i j}=C_{i j k l} \varepsilon_{k l}+R_{i j k l} w_{k l}, \quad H_{i j}=R_{k l i j} \varepsilon_{k l}+K_{i j k l} w_{k l},
$$

where $u_{i}, w_{i}$ are phonon and phason displacements, $\sigma_{i j}$ and $\varepsilon_{i j}$ phonon stresses and strains, $H_{i j}$ and $w_{i j}$ phason stresses and strains, $C_{i j k l}, K_{i j k l}$, and $R_{i j k l}$ the phonon, phason, and phonon-phason coupling elastic constants, respectively. Now we give the boundary and initial conditions; according to practical case the boundary $\Gamma$ consists of two parts $\Gamma_{t}$ and $\Gamma_{u}$; that is, $\Gamma=\Gamma_{t}+\Gamma_{u}$; at $\Gamma_{t}$ the tractions are given and at $\Gamma_{u}$ the displacements are prescribed. For the former case,

$$
\sigma_{i j} n_{j}=T_{i}, \quad H_{i j} n_{j}=h_{i}, \quad(x, y, z) \in \Gamma_{t},
$$

where $n_{j}$ represents the outward normal unit vector at any point of the boundary $\Gamma_{t}, T_{i}$ and $h_{i}$ represent the traction and generalized traction vectors, which are given functions at the boundary $\Gamma_{t}$. And for the latter case,

$$
u_{i}=\overline{u_{i}}, \quad w_{i}=\overline{w_{i}}, \quad(x, y, z) \in \Gamma_{u},
$$

where $\bar{u}_{i}$ and $\bar{w}_{i}$ are known functions at the boundary $\Gamma_{\mathcal{u}}$.

In over 200 individual QCs observed to date there is over $1 / 3$ belonging to two-dimensional decagonal QCs. So this kind of QCs is very important from fundamental point and applications. The present work is on a bending specimen with a crack and a finite width strip with a single edge crack, each of which is embedded in a two-dimensional decagonal quasicrystalline media. The complex potential theory method initially proposed by Muskhelishvili [37] is generalized to elliptic notch problem of the bending specimen. This promoted method postulated that it is valid only for rational conformal mappings. For finite width strip with a single edge crack, which belongs to an ideal plastic deformation problem, a transcendental function conformal mapping is accomplished to transform the region at the physical plane onto the upper-half plane of the mapping plane. In the first one, the complex potential functions are obtained, which can express the stresses and displacements. The stress intensity factors and the size of cohesive force zone can be derived analytically in these two problems. The practice shows that the procedure is powerful for complicated boundary value problem of the equations. Under these treatments, the original problems can be transformed into boundary value problems.

\section{Mathematical Physics Theory of Elasticity of Decagonal QCs}

First some preliminary results given using the complex potential theory will be recalled for a crack in a decagonal quasicrystalline medium.
Assume that it is perpendicular to the periodic symmetry axis (e.g., axis $z$ ); they are giving rise to two displacement fields $\mathbf{u}=\left(u_{x}, u_{y}, u_{z}\right)$ for phonon and $\mathbf{w}=\left(w_{x}, w_{y}, 0\right)$ for phason in the deformation of the decagonal QCs. Further we consider here only for plane problem; that is, it is assumed that

$$
\frac{\partial()}{\partial z}=0
$$

this leads to $\varepsilon_{z z}=\varepsilon_{x z}=\varepsilon_{y z}=0$. In addition, in the absence of body forces, the equilibrium equations are as follows:

$$
\begin{aligned}
\frac{\partial \sigma_{x x}}{\partial x}+\frac{\partial \sigma_{x y}}{\partial y}=0, & \frac{\partial \sigma_{y x}}{\partial x}+\frac{\partial \sigma_{y y}}{\partial y}=0 \\
\frac{\partial H_{x x}}{\partial x}+\frac{\partial H_{x y}}{\partial y}=0, & \frac{\partial H_{y x}}{\partial x}+\frac{\partial H_{y y}}{\partial y}=0 .
\end{aligned}
$$

When the phason field vanishes, these balance equations can be reduced to the balance equations of classical elasticity [11-15]. The explanation for readers may be seen in [38]. Equations (2), (3), and (7) are the basic equations describing elasticity of decagonal QCs under plane deformation; this is a set of 18 field equations. By using the displacement potential or stress potentials (see Fan [36]), the equation set can be reduced to a unique equation of higher order; that is, the governing equation is quadruple harmonic equation for twodimensional decagonal QCs:

$$
\nabla^{2} \nabla^{2} \nabla^{2} \nabla^{2} G=0
$$

The general solution of the quadruple harmonic equation can be written in terms of four analytic functions $g_{i}(z)(i=$ $1,2,3,4)$ of a single complex variable $z=x+i y=r e^{i \theta}$ :

$$
G=2 \operatorname{Re}\left(g_{1}(z)+\bar{z} g_{2}(z)+\frac{1}{2} \bar{z}^{2} g_{3}(z)+\frac{1}{6} \bar{z}^{3} g_{4}(z)\right)
$$

where the bar denotes the complex conjugate hereinafter; that is, $\bar{z}=x-i y=r e^{-i \theta}$. These analytic functions will be determined by boundary value conditions of practical problems. It is easy to prove that $g_{1}(z)$ has no contribution to stress and displacement fields, so we can take that

$$
g_{1}(z)=0
$$

The phonon stresses $\sigma_{i j}$, phason stresses $H_{i j}$, and the phonon displacements $u_{i}$ and phason displacements $w_{i}$ of QCs can be expressed as follows [36]:

$$
\begin{gathered}
\sigma_{x x}=-32 c_{1} \operatorname{Re}\left(\Omega(z)-2 g_{4}^{\prime \prime \prime}(z)\right), \\
\sigma_{y y}=32 c_{1} \operatorname{Re}\left(\Omega(z)+2 g_{4}^{\prime \prime \prime}(z)\right),
\end{gathered}
$$




$$
\begin{gathered}
\sigma_{x y}=\sigma_{y x}=32 c_{1} \operatorname{Im} \Omega(z), \\
H_{x x}=32 R_{1} \operatorname{Re}\left(\Theta^{\prime}(z)-\Omega(z)\right) \\
-32 R_{2} \operatorname{Im}\left(\Theta^{\prime}(z)-\Omega(z)\right), \\
H_{x y}=-32 R_{1} \operatorname{Im}\left(\Theta^{\prime}(z)+\Omega(z)\right) \\
-32 R_{2} \operatorname{Re}\left(\Theta^{\prime}(z)+\Omega(z)\right), \\
H_{y x}=-32 R_{1} \operatorname{Im}\left(\Theta^{\prime}(z)-\Omega(z)\right) \\
-32 R_{2} \operatorname{Re}\left(\Theta^{\prime}(z)-\Omega(z)\right), \\
H_{y y}=-32 R_{1} \operatorname{Re}\left(\Theta^{\prime}(z)+\Omega(z)\right) \\
+32 R_{2} \operatorname{Im}\left(\Theta^{\prime}(z)+\Omega(z)\right), \\
u_{x}+i u_{y}=32\left(4 c_{1} c_{2}-c_{3}-c_{1} c_{4}\right) g_{4}^{\prime \prime}(z) \\
-32\left(c_{1} c_{4}-c_{3}\right)\left(\overline{g_{3}^{\prime \prime \prime}(z)}+z \overline{g_{4}^{\prime \prime \prime}(z)}\right), \\
w_{x}+i w_{y}=\frac{32\left(R_{1}-i R_{2}\right)}{K_{1}-K_{2}} \overline{\Theta(z)},
\end{gathered}
$$

in which the constants are defined by

$$
\begin{gathered}
L=C_{12}, \quad M=C_{66}, \quad c=M\left(K_{1}+K_{2}\right)-2\left(R_{1}^{2}+R_{2}^{2}\right), \\
c_{1}=\frac{c}{K_{1}-K_{2}}+M, \quad c_{2}=\frac{(L+M)\left(K_{1}+K_{2}\right)+c}{4 c(L+M)}, \\
c_{3}=\frac{\left(R_{1}^{2}+R_{2}^{2}\right)}{c}, \quad c_{4}=\frac{\left(K_{1}+K_{2}\right)}{c} .
\end{gathered}
$$

Next we define new symbols for convenience [36]:

$$
\begin{gathered}
\Theta(z)=g_{2}^{(\mathrm{IV})}(z)+\bar{z} g_{3}^{(\mathrm{IV})}(z)+\frac{1}{2} \bar{z}^{2} g_{4}^{(\mathrm{IV})}(z), \\
\Omega(z)=g_{3}^{(\mathrm{IV})}(z)+\bar{z} g_{4}^{(\mathrm{IV})}(z), \\
g_{2}^{(\mathrm{IV})}(z)=h_{2}(z), \\
g_{3}^{\prime \prime \prime}(z)=h_{3}(z), \\
g_{4}^{\prime \prime}(z)=h_{4}(z),
\end{gathered}
$$

in which the prime, two primes, three primes, and superscript (IV) denote the first to fourth order differentiation to variable $z$.

In the following we consider only the stress boundary value problem; that is, the specific form of the boundary conditions at the boundary curve $L$ for decagonal QCs can be expressed by [36]

$$
\begin{aligned}
& \int\left[\sigma_{x x} \cos (\mathbf{n}, x)+\sigma_{x y} \cos (\mathbf{n}, y)\right. \\
& \left.\quad+i\left(\sigma_{y x} \cos (\mathbf{n}, x)+\sigma_{y y} \cos (\mathbf{n}, y)\right)\right] d s \\
& =\int\left(T_{x}+i T_{y}\right) d s, \\
& \int\left[H_{x x} \cos (\mathbf{n}, x)+H_{x y} \cos (\mathbf{n}, y)\right. \\
& \left.\quad+i\left(H_{y x} \cos (\mathbf{n}, x)+H_{y y} \cos (\mathbf{n}, y)\right)\right] d s \\
& =\int\left(h_{x}+i h_{y}\right) d s .
\end{aligned}
$$

Equation (14b) can be considered as the boundary conditions at the boundary curve $L$ in phason field, where $h_{x}$ and $h_{y}$ are the generalized stress in phason field. The right-hand side term of (14a) and (14b) is the resultant force of the boundary in phonon and phason fields. From (14a) and after some derivation the phonon stress boundary condition can be reduced to the equivalent form:

$$
g_{4}^{\prime \prime}(z)+\overline{g_{3}^{\prime \prime \prime}(z)}+z \overline{g_{4}^{\prime \prime \prime}(z)}=\frac{i}{32 c_{1}} \int\left(T_{x}+i T_{y}\right) d s, \quad z \in L .
$$

Meanwhile, we have the equivalent form of phason stress boundary condition:

$$
\Theta(z)=\frac{i}{R_{2}-i R_{1}} \int\left(h_{x}+i h_{y}\right) d s, \quad z \in L .
$$

Boundary conditions include both opening and shear modes (with respect to a crack); the stress intensity factors have a universal form; that is,

$$
K=K_{\mathrm{I}}-i K_{\mathrm{II}}=\frac{\sqrt{\pi}}{16 c_{1}} \lim _{\zeta \rightarrow \zeta_{1}} \frac{h_{4}^{\prime}(\zeta)}{\sqrt{\omega^{\prime \prime}(\zeta)}} .
$$

Here $K_{\mathrm{I}}$ and $K_{\mathrm{II}}$ are the "stress intensity factors," $h_{4}(\zeta)$ is the stress potential function, and $\omega(\zeta)$ is the conformal mapping corresponding to loading conditions, and $\zeta_{1}$ is located in the tip of the crack. We choose to focus on the case of uniform remote traction-free crack boundaries. This choice, although the most general, is of great interest and will serve to obtain results. In linear elasticity, many other solutions may be obtained by superposition. Thus the boundary conditions at infinity, for the two in-plane symmetry modes of fracture, are presented as

$\sigma_{x x}(\infty)=\sigma_{x y}(\infty)=0, \quad \sigma_{y y}(\infty)=0, \quad H_{i j}(\infty)=0$.

In addition, the free boundary conditions on the crack are expressed as

$$
\sigma_{x n}(s)=\sigma_{y n}(s)=0, \quad H_{x n}(s)=H_{y n}(s)=0,
$$


where $s$ is the arc-length parametrization of the crack boundary and $\mathbf{n}$ denotes the outward normal direction. In order to compute the full stress field one should first formulate the boundary conditions in terms of the analytic functions and remove the gauge freedom in (13a)-(13e). Indeed, we do not have enough boundary conditions to determine the stress potential uniquely. So we can allow in (13a)-(13e) arbitrary transformations of the form

$$
\begin{aligned}
& h_{4}(z) \longrightarrow h_{4}(z)+\mathrm{Ci} z+\gamma, \\
& h_{3}(z) \longrightarrow h_{3}(z)+\gamma^{\prime}, \\
& h_{2}(z) \longrightarrow h_{2}(z)+\gamma^{\prime \prime},
\end{aligned}
$$

where $C$ is a real constant and $\gamma, \gamma^{\prime}$, and $\gamma^{\prime \prime}$ are complex constants. This choice provides seven degrees of freedom in the definition of the stress potential. Four of them are removed according to single-valued invariance of the displacement and stress and the free boundary conditions on the crack. It is important to stress that whatever the choice of the function $h_{2}(z)$ the stresses of phonon field are unaffected, so it is not discussed. Meanwhile, the loadings on QCs considered are counterweight system. To proceed we represent $h_{3}(z)$ and $h_{4}(z)$ in Laurent expansion form:

$$
h_{4}(z)=B z+h_{4}^{0}(z), \quad h_{3}(z)=\left(B^{\prime}+i C^{\prime}\right) z+h_{3}^{0}(z)
$$

in which $B=\left(\sigma_{1}+\sigma_{2}\right) / 128 c_{1}, B^{\prime}+i C^{\prime}=-\left(\left(\sigma_{1}-\sigma_{2}\right) / 64 c_{1}\right) e^{-2 i \alpha}$, $h_{4}^{0}(z)=a_{1} / z+a_{2} / z^{2}+\cdots, h_{3}^{0}(z)=b_{1} / z+b_{2} / z^{2}+\cdots, \sigma_{1}$ and $\sigma_{2}$ are principal stresses at infinity, and $\alpha$ is the angle of $\sigma_{1}$ and $x$ axis. This form is in agreement with the boundary conditions at infinity that disallow higher order terms in $z$. Hence, the form of generalized solution using the complex potential has been established.

The existence and uniqueness of a solution to the boundary value problem originated from quasicrystal linear elasticity have been pointed out by Fan on the basis of (1)-(7); details are in [36].

\section{Boundary Value Problems of Decagonal QCs in Virtue of Dugdale's Manner}

In the linear elasticity, the stresses at the tip of a crack in a body are singular and the stresses in the neighbourhood of the crack tip may exceed the yield stress of the material constituting the body whereas inelastic deformations are nowhere permissible. To avoid this paradox, Dugdale suggested a crack model which allows the occurrence of yielding and gives an estimate for the spread of plasticity in terms of external applied loading [39]. The cohesive zone is defined as a transition region in which the surface traction smoothly changes from zero at the crack tip to a certain magnitude at the cohesive zone tip, where the displacement discontinuity across the zone disappears. The cohesive zone is a mathematical extension of the crack and can be viewed physically as the fracture process zone. Near the crack tip there is a so-called cohesive force zone whose value is unknown temporarily; this

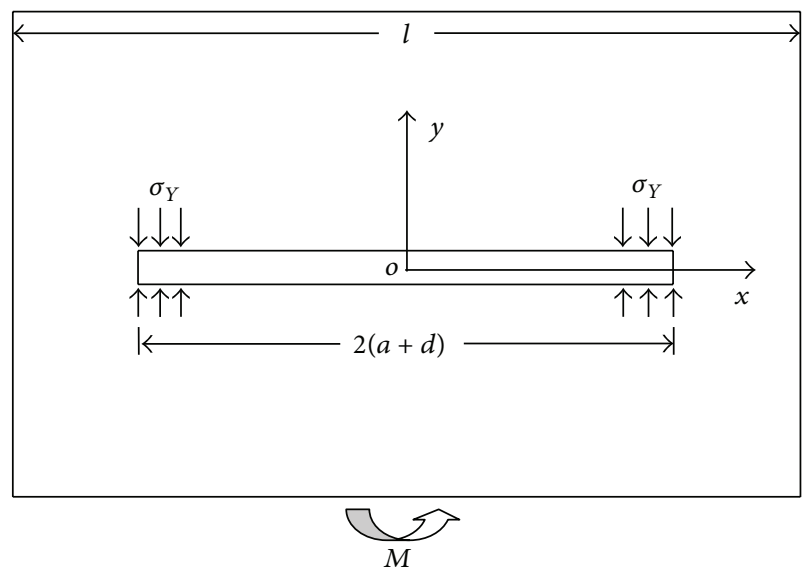

FIGURE 1: A schematic of a bending specimen weakened by a crack.

zone is subjected to stress $\sigma_{Y}$ (phonon field), in which $\sigma_{Y}$ represents the stress yield strength of the material. In essence, the Dugdale model may be regarded as a superposition of two linear elastic crack solutions: firstly, the stress field resulting from the uniform traction $p$ which tends to open the crack and secondly the surface stress over the yield zones, which is equal in magnitude to the material's yield stress $\sigma_{Y}$ and act to prevent this opening action such that the stresses are bounded at the crack tip. Based on the concept of the Dugdale model [39], the unknown strip yield zone sizes are determined by the condition of vanishing stress singularity at the crack tip; namely,

$$
K_{\mathrm{I}}^{p}+K_{\mathrm{I}}^{\sigma_{Y}}=0
$$

where $K_{\mathrm{I}}^{p}$ is the stress intensity factor of fictitious crack tip under uniform pressure stress $p$. And, $K_{I}^{\sigma_{Y}}$ represents the stress intensity factor due to the shear yield strength $\sigma_{Y}$, which can be determined by the experimental results.

\subsection{A Bending Specimen of Point Group $10 \mathrm{~mm}$ Decagonal QCs} with a Crack. Consider a bending specimen made of point group $10 \mathrm{~mm}$ decagonal QCs, weakened by a crack, as shown in Figure 1.

We do not have yet ideas of loading devices for the phason loads, so tractions in the phason field are assumed equal to zero on the boundary of a QC, from a theoretical point of view. Then the following boundary conditions should be satisfied:

$$
\begin{gathered}
|x|<\frac{l}{2}, \quad y \longrightarrow \pm \infty: h \int_{-l / 2}^{l / 2} \sigma_{y y} x d x=M \\
\sigma_{y x}=0, \quad H_{y y}=H_{y x}=0 \\
y=0: \sigma_{y y}=\sigma_{y x}=0, \quad H_{y y}=H_{y x}=0 \\
\begin{array}{c}
a<\mid<a, \quad \\
\sigma_{y x}=0, \quad H_{y y}=H_{y x}=0
\end{array}
\end{gathered}
$$




$$
\begin{gathered}
|x|=\frac{l}{2}, \quad-\infty<y<\infty: \sigma_{x x}=\sigma_{x y}=0, \\
H_{x x}=H_{x y}=0
\end{gathered}
$$

in which $M$ denotes the bending moment subjected to the upper and lower surface of the specimen, and $h$ is the thickness and $l$ the width of the specimen. We assume that the plastic zone around crack tip is of a narrow band type, with length denoted by $d$ : its value to be determined. The difficulty of the problem at hands rests essentially on the complicated boundary conditions. The exact analytic solution is not available. We here can obtain an approximate solution. For this purpose we decompose the problem into two secondary problems, one corresponding to the boundary conditions:

$$
\begin{gathered}
|x|<\frac{l}{2}, \quad y \longrightarrow \pm \infty: h \int_{-l / 2}^{l / 2} \sigma_{y y} x d x=M, \\
\sigma_{y x}=0, \quad H_{y y}=H_{y x}=0 ; \\
|x|<a+d, \quad y=0: \sigma_{y y}=0, \quad \sigma_{y x}=0, \\
H_{y y}=H_{y x}=0 ; \\
-\infty<x^{2}, \quad y<\sigma_{x x}=\sigma_{x y}=0, \\
H_{x x}=H_{x y}=0,
\end{gathered}
$$

and the other is related to the boundary conditions:

$$
\begin{aligned}
|x|<\frac{l}{2}, & y \longrightarrow \pm \infty: \sigma_{y y}=\sigma_{y x}=0, \\
H_{y y} & =H_{y x}=0 ; \\
y=0: \sigma_{y y}=0, \quad \sigma_{y x}=0, & \\
H_{y y} & =H_{y x}=0 ; \\
y<|x|<a+d, & =0: \sigma_{y y}=\sigma_{Y}, \quad \sigma_{y x}=0, \\
H_{y y} & =H_{y x}=0 ; \\
-\infty & <y<\infty: \sigma_{x x}=\sigma_{x y}=0, \\
H_{x x} & =H_{x y}=0 .
\end{aligned}
$$

The boundary value problem (8) ((23a)-(23c)) is a pure bending problem of cracked specimen. Muskhelishvili studied a bending specimen with an elliptic notch, but the major axis of the ellipse is parallel to the $z$-axis; the solution is no usage to the present problem; the methodology of Muskhelishvili is still effective when the condition $2 a / l \leq 1 / 3$ is satisfied [37]. By making these simplifications, the boundary value problems also cannot be solved at the physical plane (i.e., the $z$-plane). We need to employ a conformal mapping to transform the problem for solving at mapping plane. Here we must take an approximation and assume that the size of crack is quite smaller than that of the specimen, and we can use the conformal mapping

$$
z=\omega(\zeta)=\frac{a+d}{2}\left(\frac{1}{\zeta}+\zeta\right)
$$

to transform the region of the specimen onto the interior of the unit circle at the $\zeta$-plane; we find the approximate solution at the conformal mapping plane by solving (15a) and the boundary conditions (23a)-(23c) such as

$$
h_{4}(\zeta)=\frac{1}{32 c_{1}}\left\{\frac{3 M(a+d)^{2}}{2 h l^{3}} \zeta^{2}+\frac{3 M(a+d)^{2}}{h l^{3}}\right\} .
$$

In the equation some terms being independent from crack stress state are not included.

The result related to the boundary value problem (24a)(24d) is obtained; then,

$$
\begin{aligned}
\tilde{h}_{4}(\zeta)=\frac{1}{32 c_{1}}\{ & \frac{\sigma_{Y}(a+d) \varphi_{2}}{\pi} \frac{1}{\zeta} \\
& \left.-\frac{\sigma_{Y}}{2 \pi i}\left[\ln \frac{\zeta^{2}-\sigma}{\zeta^{2}-\bar{\sigma}}-a \ln \frac{\left(\zeta-\sigma_{2}\right)\left(\zeta+\sigma_{2}\right)}{\left(\zeta+\bar{\sigma}_{2}\right)\left(\zeta-\bar{\sigma}_{2}\right)}\right]\right\},
\end{aligned}
$$

where $\sigma=\mathrm{e}^{i \varphi}$ represents the value of $\zeta$ corresponding point at the unit circle $\gamma, \bar{\sigma}=\mathrm{e}^{-i \varphi}$ represents the conjugate of $\sigma$, and $\sigma_{2}=\mathbf{e}^{i \varphi_{2}}$ corresponds to the point $z=a$ at the physical plane and $\bar{\sigma}_{2}=\mathrm{e}^{-i \varphi_{2}}$ represents the conjugate of $\sigma_{2}$. It is evident that $\cos \varphi_{2}=a /(a+d)$ and $\sigma_{2}+1 / \sigma_{2}=2 \cos \varphi_{2}$. The solution (27) is constructed for the infinite plane with a Dugdale crack, which approximately holds for the specimen with finite width l. Since the complex analysis is well known, the detail of above calculation is omitted. In light of (26) and (25), meanwhile noting that (16), we have

$$
K_{\mathrm{I}}^{(1)}=6 \frac{M(a+d)}{h l^{3}} \sqrt{\pi(a+d)} .
$$

From (27) and (25) one finds that

$$
K_{\mathrm{I}}^{(2)}=-2 \sigma_{Y} \sqrt{\frac{a+d}{\pi}} \arccos \left(\frac{a}{a+d}\right) .
$$

Because at the tip of the cohesive force zone there is no stress singularity; that is, the stress intensity factor at the point should be zero; that is, the requirement of (21) leads to

$$
\frac{3 M(a+d) \pi}{h l^{3}}=\sigma_{Y} \arccos \left(\frac{a}{a+d}\right) .
$$

The size $d$ of the plastic zone is determined already, though the relation is a nonlinear equation obviously. Due to $d \ll$ $a$ and $2 a \ll l$, the formula (30) is highly approximated. To obtain the stresses and displacements, the solution of $h_{4}(\zeta)$ and $h_{3}(\zeta)$ is enough. All can be determined on basis of (11a)(11i) and (13a)-(13e). 


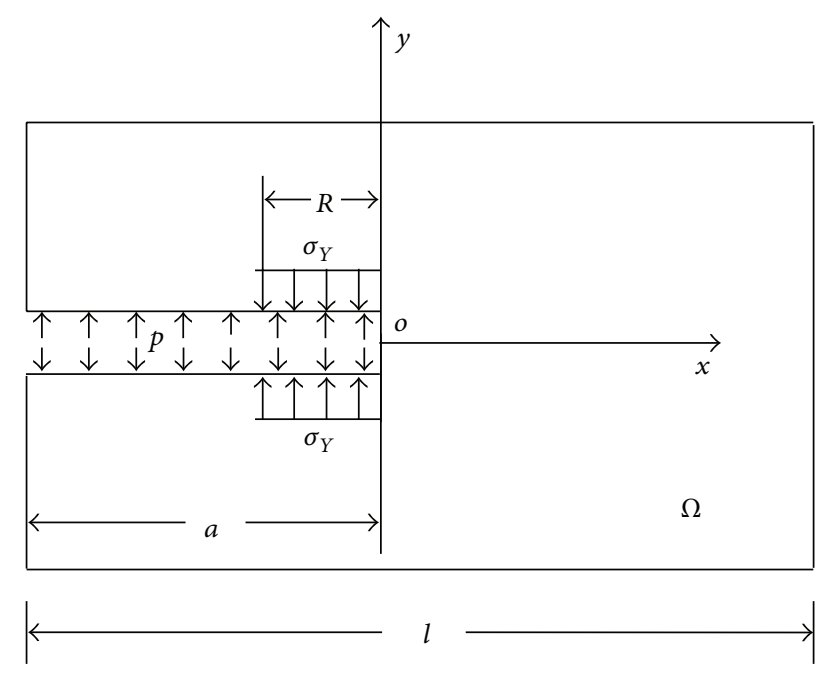

FIGURE 2: A schematic of the crack containing a cohesive zone ahead of the crack tip.

3.2. A Finite Width Strip of Decagonal QCs with a Single Edge Crack. Consider a strip with a single crack embedded in decagonal QCs, in which $l$ stands for the width. Figure 2 shows a scheme of the crack containing a cohesive zone ahead of the crack tip.

A transcendental function conformal mapping constructed corresponding to the transformation between the region $\Omega$ in the physical plane and the upper half-plane in $\zeta$-plane reads

$$
z=\omega(\zeta)=\frac{2 l}{\pi} \arctan \left[\tan \left(\frac{\pi a}{2 l}\right) \sqrt{1-\zeta^{2}}\right]-a .
$$

It can transform the cracked material shown in Figure 1 onto the upper-half plane of the mapping plane. Under the mapping (31), the crack tip $z=0$ is mapped to $\zeta=0$, while $z=\left(-a, 0^{ \pm}\right)$are mapped to $\zeta= \pm 1$. As shown in Figure 2 , the boundary conditions of this problem of QCs can be written as follows:

$$
\begin{gathered}
-a<x<l-a, \quad y \longrightarrow \pm \infty: \sigma_{y y}=\sigma_{y x}=0 \\
H_{y y}=H_{y x}=0 ; \\
x=l-a-\infty<y<\infty: \sigma_{x x}=\sigma_{x y}=0 \\
H_{x x}=H_{x y}=0 ; \\
-a<x<0, \quad y= \pm 0: \sigma_{y y}=-p, \quad \sigma_{x y}=0 \\
H_{y y}=H_{x y}=0 \\
\quad y \longrightarrow \pm \infty: \sigma_{y y}=\sigma_{y x}=0 \\
-a<x<l-a, \quad y \\
H_{y y}=H_{y x}=0 ;
\end{gathered}
$$

$$
\begin{gathered}
x=-a, \quad x=l-a-\infty<y<\infty: \sigma_{x x}=\sigma_{x y}=0, \\
H_{x x}=H_{x y}=0 ; \\
-a<x<0, \quad y= \pm 0: \sigma_{y y}=\sigma_{Y}, \quad \sigma_{x y}=0, \\
H_{y y}=H_{x y}=0 .
\end{gathered}
$$

Meanwhile, based on the above figure of a finite width strip with single edge crack of two-dimensional QCs and the boundary conditions, the functional equations can be established by means of (15a) and its conjugated equation:

$$
\begin{aligned}
& h_{4}(\zeta)+\frac{1}{2 \pi i} \int_{\gamma} \frac{\omega(\sigma)}{\overline{\omega^{\prime}(\sigma)}} \frac{\overline{h_{4}^{\prime}(\sigma)}}{\sigma-\zeta} d \sigma+\overline{h_{3}(0)}=\frac{1}{2 \pi i} \int_{\gamma} \frac{f_{0}}{\sigma-\zeta} d \sigma, \\
& \overline{h_{4}(0)}+\frac{1}{2 \pi i} \int_{\gamma} \frac{\overline{\omega(\sigma)}}{\omega^{\prime}(\sigma)} \frac{h_{4}^{\prime}(\sigma)}{\sigma-\zeta} d \sigma+h_{3}(\zeta)=\frac{1}{2 \pi i} \int_{\gamma} \frac{\bar{f}_{0}}{\sigma-\zeta} d \sigma,
\end{aligned}
$$

in which $\sigma$ represents the value of $\zeta$ at the real axis $\gamma$ in the mapping plane, and

$$
\begin{aligned}
f_{0}(z) & =\frac{i}{32 c_{1}} \int_{-a}^{z}\left(T_{x}+i T_{y}\right) d s \\
& =-\frac{1}{32 c_{1}} \int_{-a}^{z} p d z \\
& = \begin{cases}-\frac{1}{32 c_{1}} p(z+a) & -a<x<0 \\
0 & \text { other } z \text { on } \partial \Omega .\end{cases}
\end{aligned}
$$

By solving the foregoing equations, one gets the result

$$
h_{4}^{\prime}(\zeta)=-\frac{1}{32 c_{1}} \frac{1}{2 \pi i} \int_{-1}^{1} \frac{p \omega^{\prime}(\sigma)}{\sigma-\zeta} d \sigma
$$

Finally, substituting (36) and (31) into (16) yields the exact stress intensity factors:

$$
K_{\mathrm{I}}=p \sqrt{2 l \tan \left(\frac{\pi a}{2 l}\right)}, \quad K_{\mathrm{II}}=0 .
$$

Similarly, if the uniform pressure on the surface of the crack is replaced by a shear force $\tau$, we can find the stress intensity factors:

$$
K_{\mathrm{I}}=0, \quad K_{\mathrm{II}}=\tau \sqrt{2 l \tan \left(\frac{\pi a}{2 l}\right)} .
$$

The stress intensity factor due to the yield strength $\sigma_{Y}$ can be expressed by

$$
K_{\mathrm{I}}^{\sigma_{Y}}=\frac{\sqrt{\pi}}{16 c_{1}} \frac{{\widetilde{h_{4}}}^{\prime}(0)}{\sqrt{\omega^{\prime \prime}(0)}}
$$

in which $\widetilde{h_{4}}(0)$ can be determined like $h_{4}^{\prime}(0)$. In the same way the requirement of (21) leads to the size of cohesive force zone:

$$
\pi(a-R)=2 l \arctan \left(\frac{1}{\sqrt{A^{2}+B^{2}}}\right),
$$


in which

$$
A=\frac{1}{\tan (\pi a / 2 l)}, \quad B=\frac{\tan \left(p \pi / 2 \sigma_{Y}\right)}{\sin (\pi a / 2 l)} .
$$

\section{Conclusion and Discussion}

This work employs the complex potentials and conformal mapping techniques to analyze the equilibrium boundary value problems for elastic quasicrystals. In virtue of the generalized solutions, stress and displacement boundary value problems of decagonal QCs are transferred to boundary value problems of analytic functions in a given region. Two separated problems emerge and can be analyzed. The stress intensity factors can be expressed in exact analytic forms. Although there exists phason field in QCs, (28), (29) and (37), (38) show that the intensity factors of phonon and phason fields are independent of material constants. Since both phonon and phason stress and displacement fields are dependent on all of the material constants, this result indicates the important effect of phason and phonon-phason coupling to the fracture parameters. The Dugdale model is a simple and efficient method to study the ideal elastic-plastic cracks. The results of these problems studied in this paper show that phason field and phonon-phason coupling have no effect on the plastic zone size, which is useful for plastic deformation of decagonal QCs. All the results can be reduced to the corresponding results of linear elastic theory when the phason elastic constants are approaching to zero. This work reveals the physical sense of the results relative to phason and the difference between the mechanical behaviors of the crack problem in crystals and decagonal QCs. It is presented that the mechanic problems can be transferred to boundary value problems of analytic functions.

\section{Conflict of Interests}

The authors declare that there is no conflict of interests regarding the publication of this paper.

\section{Acknowledgments}

This work is supported by the National Natural Science Foundation of China (Grant no. 11402158) and the Qualified Personnel Foundation of Taiyuan University of Technology (Grant no. tyut-rc201358a).

\section{References}

[1] D. Shechtman, I. Blech, D. Gratias, and J. W. Cahn, "Metallic phase with long-range orientational order and no translational symmetry," Physical Review Letters, vol. 53, no. 20, pp. 1951-1953, 1984.

[2] S. Fischer, A. Exner, K. Zielske et al., "Colloidal quasicrystals with 12 -fold and 18-fold diffraction symmetry," Proceedings of the National Academy of Sciences of the United States of America, vol. 108, no. 5, pp. 1810-1814, 2011.

[3] R. Mikulla, J. Stadler, and H. R. Trebin, "Crack propagation in quasicrystals," Physical Review Letters, vol. 81, pp. 3163-3166, 1998.
[4] J. Bohsung and H. R. Trebin, "Disclinations in quasicrystals," Physical Review Letters, vol. 58, no. 12, pp. 1204-1207, 1987.

[5] K. Edagawa, "Phonon-phason coupling in decagonal quasicrystals," Philosophical Magazine, vol. 87, no. 18-21, pp. 2789-2798, 2007.

[6] K. Edagawa, Dislocation in Solids, Elsevier, Amsterdam, The Netherlands, 2007.

[7] C. Walz, Zur Hydrodynamik in Quasikristallen, Diplomarbeit, Universitaet Stuttgart, 2003.

[8] H. Lipp, M. Engel, S. Sonntag, and H.-R. Trebin, "Phason dynamics in one-dimensional lattices," Physical Review B, vol. 81, no. 6, Article ID 064302, 2010.

[9] M. Engel, M. Umezaki, H.-R. Trebin, and T. Odagaki, "Dynamics of particle flips in two-dimensional quasicrystals," Physical Review B-Condensed Matter and Materials Physics, vol. 82, no. 13, Article ID 134206, 2010.

[10] F. Rösch, C. Rudhart, J. Roth, H.-R. Trebin, and P. Gumbsch, "Dynamic fracture of icosahedral model quasicrystals: a molecular dynamics study," Physical Review B, vol. 72, no. 1, Article ID 014128, 2005.

[11] P. Bak, "Phenomenological theory of icosahedral incommensurate ("Quasiperiodic") order in Mn-Al Alloys," Physical Review Letters, vol. 54, no. 14, pp. 1517-1519, 1985.

[12] P. Bak, "Symmetry, stability, and elastic properties of icosahedral incommensurate crystals," Physical Review B: Condensed Matter. Third Series, vol. 32, no. 9, pp. 5764-5772, 1985.

[13] T. C. Lubensky, S. Ramaswamy, and J. Toner, "Hydrodynamics of icosahedral quasicrystals," Physical Review B, vol. 32, no. 11, pp. 7444-7452, 1985.

[14] D.-H. Ding, W. Yang, C. Hu, and R. Wang, "Generalized elasticity theory of quasicrystals," Physical Review B, vol. 48, no. 10, pp. 7003-7010, 1993.

[15] C. Hu, R. Wang, and D. Ding, "Symmetry groups, physical property tensors, elasticity and dislocations in quasicrystals," Reports on Progress in Physics, vol. 63, no. 1, pp. 1-39, 2000.

[16] W. Li and T. Y. Fan, "Dynamic analysis of cracked octagonal quasicrystals," Mathematical Problems in Engineering, vol. 2011, Article ID 567489, 18 pages, 2011.

[17] S. Francoual, F. Livet, M. De Boissieu et al., "Dynamics of phason fluctuations in the i-AlPdMn quasicrystal," Physical Review Letters, vol. 91, Article ID 225501, 2003.

[18] G. Coddens, "On the problem of the relation between phason elasticity and phason dynamics in quasicrystals," European Physical Journal B, vol. 54, no. 1, pp. 37-65, 2006.

[19] J. B. Wang, L. Mancini, R. H. Wang, and J. Gastaldi, "Phononand phason-type spherical inclusions in icosahedral quasicrystals," Journal of Physics Condensed Matter, vol. 15, no. 24, pp. L363-L370, 2003.

[20] M. Feuerbacher, M. Bartsch, B. Grushko, U. Messerschmidt, and K. Urban, "Plastic deformation of decagonal Al-Ni-Co quasicrystals," Philosophical Magazine Letters, vol. 76, no. 6, pp. 369-376, 1997.

[21] M. Feuerbacher, B. Baufeld, R. Rosefeld et al., "Intrinsic deformation properties of icosahedral Al-Pd-Mn single quasicrystals," Philosophical Magazine Letters, vol. 71, pp. 91-98, 1995.

[22] M. Wollgarten, M. Beyss, K. Urban, H. Liebertz, and U. Köster, "Direct evidence for plastic deformation of quasicrystals by means of a dislocation mechanism," Physical Review Letters, vol. 71, no. 4, pp. 549-552, 1993. 
[23] R. Rosenfeld and M. Feuerbacher, "Study of plastically deformed icosahedral Al-Pd-Mn single quasicrystals by transmission electron microscopy," Philosophical Magazine Letters, vol. 72, pp. 375-784, 1995.

[24] P. Schall, M. Feubrbacher, M. Bartsch, U. Messerschmidt, and K. Urban, "Dislocation density evolution upon plastic deformation of Al-Pd-Mn single quasicrystals," Philosophical Magazine Letters, vol. 79, no. 10, pp. 785-796, 1999.

[25] B. Geyer, M. Bartsch, M. Feuerbacher, K. Urban, and U. Messerschmidt, "Plastic deformation of icosahedral Al-PdMn single quasicrystals I. Experimental results," Philosophical Magazine A, vol. 80, no. 5, pp. 1151-1163, 2000.

[26] U. Messerschmidt, M. Bartsch, M. Feuerbacher, B. Geyer, and K. Urban, "Friction mechanism of dislocation motion in icosahedral Al-Pd-Mn quasicrystals," Philosophical Magazine A, vol. 79, no. 9, pp. 2123-2135, 1999.

[27] P. Ebert, M. Feuerbacher, N. Tamura, M. Wollgarten, and K. Urban, "Evidence for a cluster-based structure of AIPdMn single quasicrystals," Physical Review Letters, vol. 77, no. 18, pp. 3827-3830, 1996.

[28] H. Takakura, M. Shiono, T. J. Sato, A. Yamamoto, and A. P. Tsai, "Ab initio structure determination of icosahedral $\mathrm{Zn}-\mathrm{Mg}$-Ho quasicrystals by density modification method," Physical Review Letters, vol. 86, no. 2, pp. 236-239, 2001.

[29] D. Caillard, G. Vanderschaeve, L. Bresson, and D. Gratias, "Transmission electron microscopy study of dislocations and extended defects in as-grown icosahedral Al-Pd-Mn single grains," Philosophical Magazine A, vol. 80, no. 1, pp. 237-253, 2000.

[30] X. Y. Li and P. D. Li, "Three-dimensional thermo-elastic general solutions of one-dimensional hexagonal quasi-crystal and fundamental solutions," Physics Letters A: General, Atomic and Solid State Physics, vol. 376, no. 26-27, pp. 2004-2009, 2012.

[31] X.-Y. Li, "Fundamental solutions of penny-shaped and halfinfinite plane cracks embedded in an infinite space of onedimensional hexagonal quasi-crystal under thermal loading," Proceedings of the Royal Society A: Mathematical, Physical and Engineering Sciences, vol. 469, no. 2154, Article ID 20130023, 2013.

[32] E. Radi and P. M. Mariano, "Stationary straight cracks in quasicrystals," International Journal of Fracture, vol. 166, pp. 102-120, 2010.

[33] E. Radi and P. M. Mariano, "Steady-state propagation of dislocations in quasi-crystals," Proceedings of The Royal Society of London A, vol. 467, no. 2136, pp. 3490-3508, 2011.

[34] L. H. Li and G. T. Liu, "Stroh formalism for icosahedral quasicrystal and its application," Physics Letters A, vol. 376, no. 8-9, pp. 987-990, 2012.

[35] J. H. Guo, J. Yu, and Y. M. Xing, "Anti-plane analysis on a finite crack in a one-dimensional hexagonal quasicrystal strip," Mechanics Research Communications, vol. 52, pp. 40-45, 2013.

[36] T. Y. Fan, Mathematical Theory of Elasticity of Quasicrystals and Its Applications, Springer, Heideberg, Germany, 2010.

[37] N. I. Muskhelishvili, Some Basic Problems of Mathematical Theory of Elasticity, Noordhoff, Groningen, The Netherlands, 1963.

[38] P. M. Mariano and J. Planas, "Phason self-actions in quasicrystals," Physica D, vol. 249, pp. 46-57, 2013.

[39] D. S. Dugdale, "Yielding of steel sheets containing slits," Journal of the Mechanics and Physics of Solids, vol. 8, no. 2, pp. 100-104, 1960. 


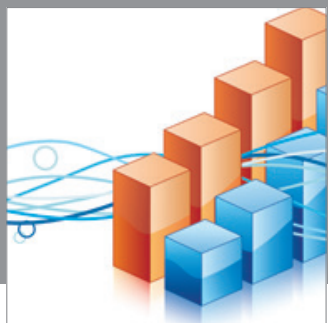

Advances in

Operations Research

mansans

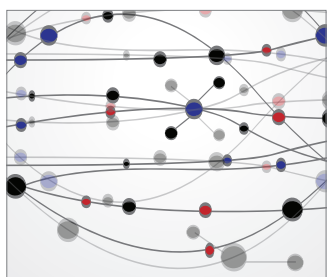

The Scientific World Journal
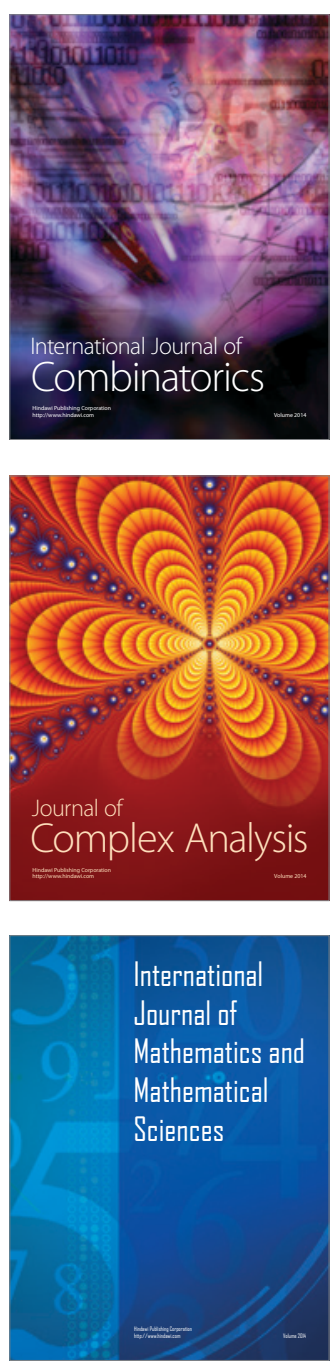
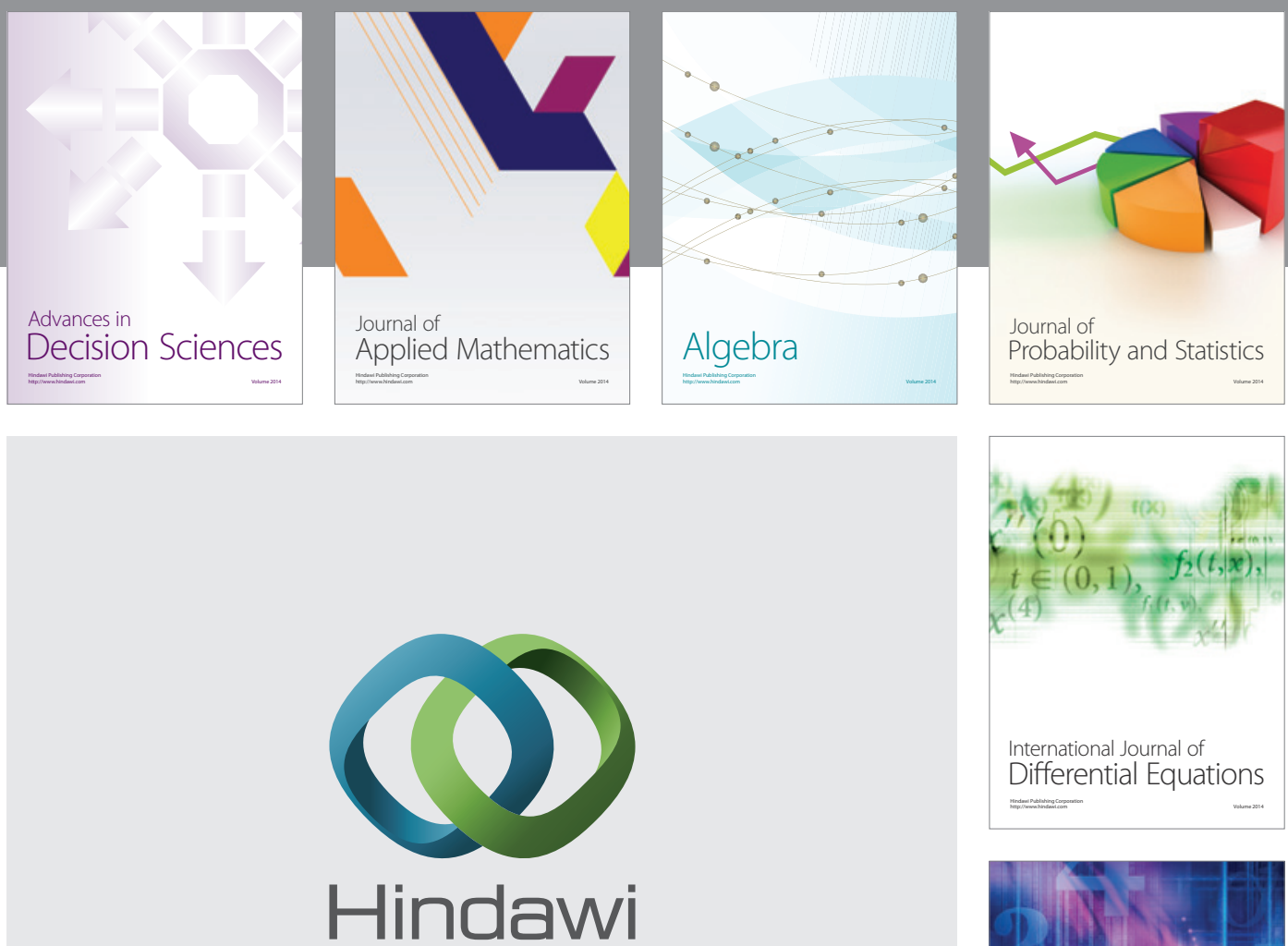

Submit your manuscripts at http://www.hindawi.com
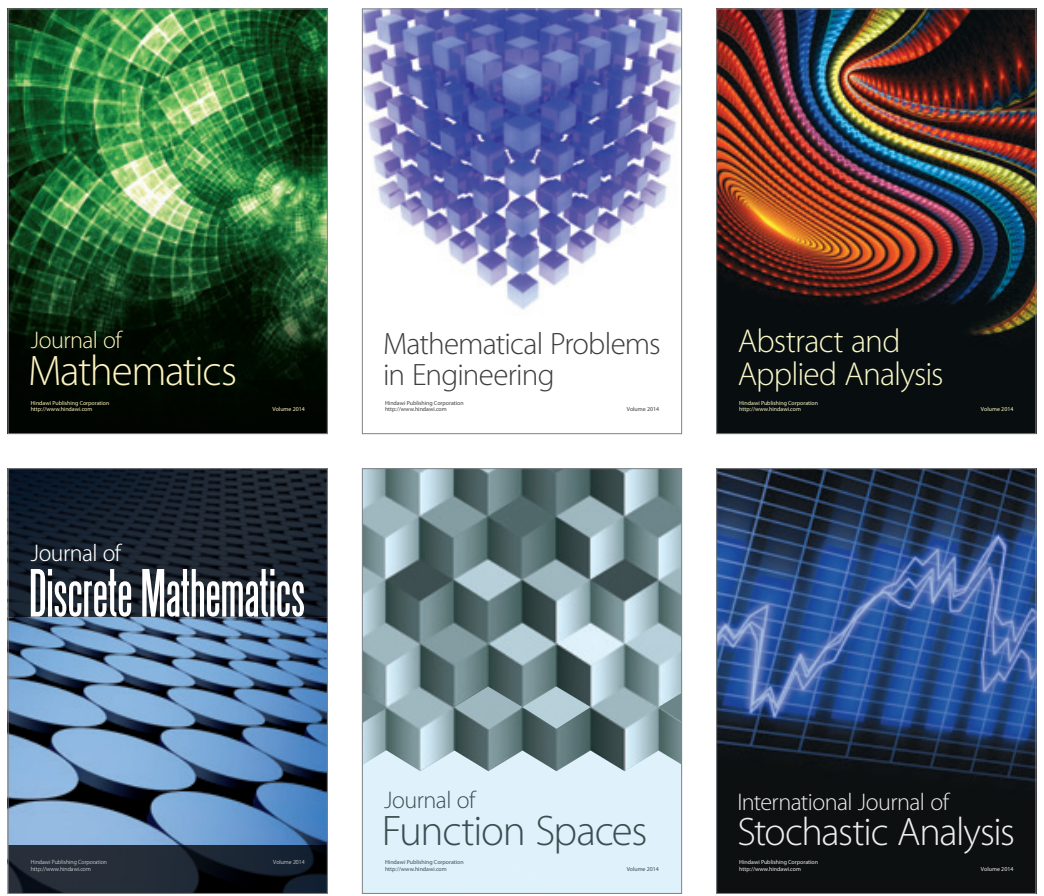

Journal of

Function Spaces

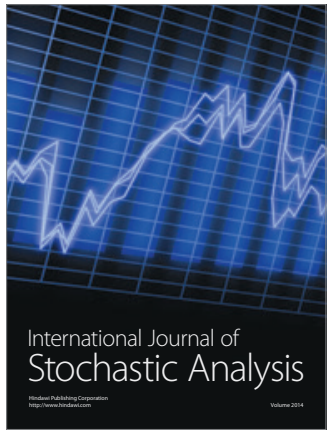

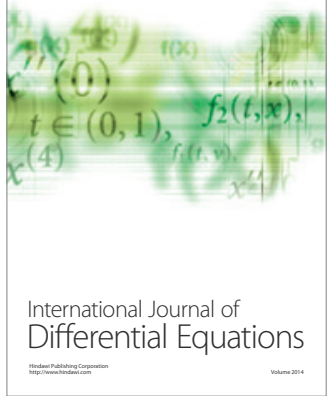
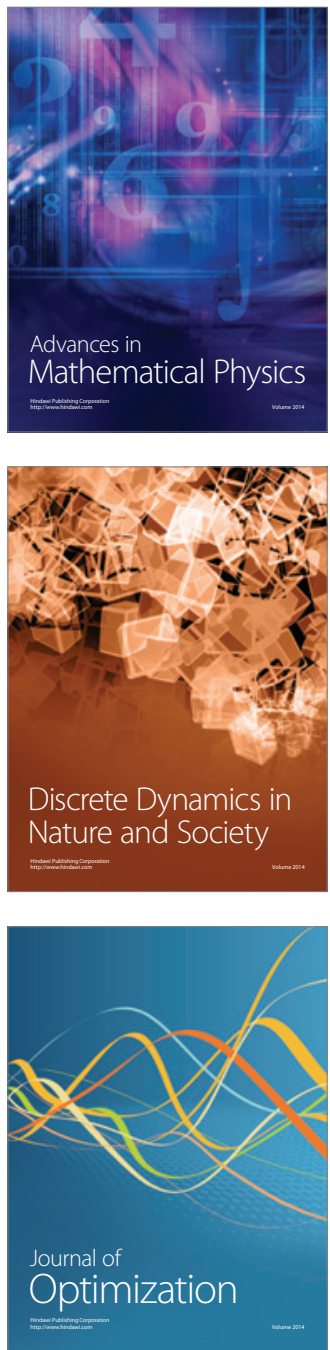